\title{
Viscoelastic effects during unloading in depth-sensing indentation
}

\author{
A.H.W. Ngan and B. Tang \\ Department of Mechanical Engineering, The University of Hong Kong, Pokfulam Road, \\ Hong Kong, People's Republic of China
}

(Received 9 April 2002; accepted 11 July 2002)

\begin{abstract}
With polypropylene as a prototype viscoelastic material at room temperature, it was found that a "nose" may appear in the unloading segment of the load-displacement curve during nanoindentation when the holding time at peak load is short and/or the unloading rate is small, and when the peak load is high enough. The load at which the nose appears was also found to decrease linearly with decreasing unloading rate. A linear viscoelasticity analysis was performed to interpret this effect. The analysis predicts a linear variation between the nose load and the unloading rate, and the slope of such a linear variation is also shown to be proportional to the viscosity parameter of the material. Thus, by measuring the slope of the nose-load versus unloading rate plot at a given temperature, the viscosity parameter of the specimen can be found. This is a new way of measuring the viscosity parameter of a material in addition to the existing method of force modulation and noting the frequency response of the displacement.
\end{abstract}

\section{INTRODUCTION}

In the past decade nanoindentation has been established as a standard method for probing mechanical properties including hardness and elastic modulus at the submicron length scale. In the celebrated method for elastic modulus measurement by Oliver and Pharr, ${ }^{1}$ the tip-sample contact stiffness $S$ and contact area $A_{\mathrm{c}}$ at the onset of unload are measured. The tip-sample reduced modulus $E_{\mathrm{r}}$ is then calculated from the contact mechanics relation:

$$
E_{\mathrm{r}}=\frac{\sqrt{\pi}}{2} \frac{S}{\sqrt{A_{\mathrm{c}}}} .
$$

Here, $A_{\mathrm{c}}$ is the contact area at full load and is therefore not the residual indent area after unload. This is particularly important for materials like glass or polymers, in which the residual indent size after unload could be significantly smaller than the contact size at full load. Thus, the requirement for area measurement in hardness and elastic modulus measurement is different. Since hardness is defined as the load divided by the residual indent area, we are more concerned with the residual indent area after unload, which can be measured by atomic force microscopy (AFM), for example. This is the prime reason for incorporating AFM on the same platform as indentation in the newest nanoindenters such as the Hysitron (Hysitron Inc., Minneapolis, MN) or the CSM (CSM Instruments SA, Peseux, Switzerland) setups. In elastic modulus measurement, currently the only way of measuring the contact area $A_{\mathrm{c}}$ under load is to use the area-depth function $A_{\mathrm{c}}=f\left(h_{\mathrm{c}}\right)$ of the tip, and the contact depth $h_{\mathrm{c}}$ (see Fig. 1) is obtained using the Oliver-Pharr formula:

$$
h_{\mathrm{c}}=h-\epsilon \frac{P}{S},
$$

where $\epsilon$ is a constant depending on the indenter geometry ( $\epsilon=0.75$ for the Berkovich tip).

Equations (1) and (2) are purely results of elastic contact mechanics. In nanoindentation, it is often found that significant viscoelastic effects in general are present at full load. A consequence of pure elasticity is that the contact size or indenter displacement will decrease spontaneously as load decreases. When viscosity effects dominate, however, the indenter displacement can actually increase for a short time, even though the load has started to decrease from the full load value at the onset of unload. The resultant load-displacement plot in this case will exhibit a "nose" in the unloading segment. When a nose occurs, the apparent unloading contact stiffness $S$, defined as $\mathrm{d} P / \mathrm{d} h$ (see Fig. 1), is negative and the reduced modulus can no longer be calculated from Eq. (1). Even when a conspicuous nose does not occur, the presence of viscosity may lead to overestimation of the contact stiffness and hence the elastic modulus of the sample.

In a recent paper, Feng and Ngan ${ }^{2,3}$ proposed a method for correcting viscoelastic effects in the measurement of the unloading contact stiffness using Eq. (1). The correction term in the contact compliance $1 / S$ was found to be, at least in the case of linear viscoelasticity, 


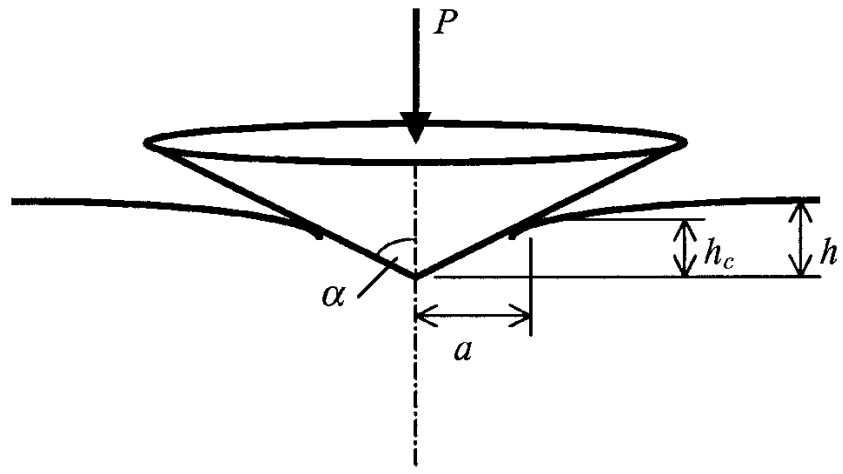

FIG. 1. Conical indentation into a half-space.

$\dot{h}_{\mathrm{h}} / \dot{P}_{\mathrm{u}}$, where $\dot{h}_{\mathrm{h}}$ is the displacement drift rate, due to any combination between sample viscosity and machine drifts, at the end of the load hold prior to unload, and $\dot{P}_{\mathrm{u}}$ is the unloading rate at the onset of unload. So far, no correction for viscoelastic effects has been proposed for the measurement of the contact area $A_{\mathrm{c}}$ from Eq. (2).

This paper explores the extension of the concepts developed by Feng and Ngan, ${ }^{2,3}$ and focuses on the conditions under which a nose will appear in the unloading portion of the load-displacement curve in a viscoelastic material. We believe that understanding viscoelastic behavior during indentation is a timely research topic because high-temperature indenters are being actively developed by the various suppliers, and hence in the near future, creep properties at the submicron length scale can be intensively investigated by high-temperature nanoindentation. Although our present experiments were carried out on an ambient temperature indenter, the samples we used were polypropylene, for which ambient temperature is already a high temperature compared to the melting/glass transition temperature of the material. We believe that the viscoelastic indentation behavior discussed below is likely to be generic at temperatures high relative to the melting temperature.

\section{EXPERIMENTAL}

The indentation experiments reported below were carried out on polypropylene (PP) slabs with dimensions of about $2 \mathrm{~cm} \times 2 \mathrm{~cm} \times 3 \mathrm{~mm}$. The PP samples were polished down to $1 \mu \mathrm{m}$ prior to indentation. Indentation experiments were carried out at room temperature using a commercial nanoindenter (CSM Instruments SA, Peseux, Switzerland) with a Berkovich tip. The nanoindenter has a maximum load of $300 \mathrm{mN}$. In the load schedules employed, the load was first ramped up to a selected peak value at a constant loading rate. This is followed by a period of holding at the peak load, followed by subsequent unload at a constant rate.

Our CSM Instruments indentator is equipped with a "sapphire-ring" design in which a sapphire ring encircling the indenter tip is pressed lightly onto the sample surface during indentation. The displacement of this sapphire ring and hence the sample surface is recorded, together with that of the tip during indentation, and the tip displacement output by the machine is the relative displacement between the two. This design therefore helps to reduce the effect of thermal drift, as any drifts of the tip and sample together will be subtracted out. Our CSM Instruments indenter is placed side-by-side a Hysitron $8 \mathrm{mN}$-indenter, mounted onto a Thermomicroscopes (TM Microscopes, Sunnyvale, CA) scanning probe microscope. This arrangement allows us to compare simultaneously the drift characteristics of both machines. In a series of low-load hold experiments on identical samples, we found that the typical drift rate of the Hysitron ${ }^{\circledR}$ machine was about $0.01 \mathrm{~nm} / \mathrm{s}$, while that of the CSM machine was about $0.1 \mathrm{~nm} / \mathrm{s}$.

The indentation experiments reported below were typically completed in less than $30 \mathrm{~min}$, and at a peak load of, say, $200 \mathrm{mN}$, the maximum indenter displacement of PP is about $10 \mu \mathrm{m}$. With a typical drift rate of $0.1 \mathrm{~nm} / \mathrm{s}$, the maximum thermal drift in our experiments would be less than $0.18 \mu \mathrm{m}$, which is about $1.8 \%$ of the maximum displacement in PP at $200 \mathrm{mN}$. Since the thermal drift is so small compared to the indent depth, no drift correction was applied to the results reported below. We verified, in a few typical sets of results, that inclusion of thermal drift rate did not change the qualitative appearance of the load-displacement curves.

Since our indents were large, we did not find severe variations of the results over the surface of the samples. Occasionally, probably due to microstructural homogeneities, significant deviations were observed in the loading curves of some indents upon repeated testing using the same test conditions, and these results were discarded.

\section{RESULTS}

Figure 2 shows the load-displacement $(P-h)$ curves of PP in three independent experiments with the same loading rate and hold time at peak load but with different unloading rates. It can be seen that when the unloading rate is fast $(8.333 \mathrm{mN} / \mathrm{s})$, the unloading segment of the $P$ - $h$ curve looks elastic without the appearance of a nose. However, as the unloading rate decreases to $1.667 \mathrm{mN} / \mathrm{s}$, the displacement can be seen to continue to increase briefly after the onset of unload, and it passes through a continuous maximum at a load of $184 \mathrm{mN}$ before decreasing as the load drops further. In other words, a nose in the unloading $P$ - $h$ curve occurred. When the unloading rate is further decreased to $0.167 \mathrm{mN} / \mathrm{s}$, the nose is even more conspicuous. Also, the load at which the nose appears drops to $180 \mathrm{mN}$.

The load $P$ at which a nose appears in the unloading $P$ - $h$ curve, denoted as $P_{\mathrm{n}}$ hereafter, was found to be dependent on both the unloading rate and the holding time 
before unload. Figure 3 shows the dependence of $P_{\mathrm{n}}$ on the unloading rate in a series of independent indentations to the same peak load of $200 \mathrm{mN}$ using the same loading rate of $13.33 \mathrm{mN} / \mathrm{s}$ but different holding times before unload. It can be seen that at each holding time, $P_{\mathrm{n}}$ decreases linearly with reduction in the unloading rate. It is also evident from Fig. 3 that the slope of the $P_{\mathrm{n}}$ versus unloading rate at each holding time exhibits a clear increasing trend with holding time.

Figure 4 shows the $P_{\mathrm{n}}$ versus unloading rate plots at different peak loads, while the loading rate is kept constant at $13.33 \mathrm{mN} / \mathrm{s}$, and the holding time at peak load is also held constant at $8 \mathrm{~s}$. It can be seen that at each peak load, $P_{\mathrm{n}}$ varies linearly with the unloading rate, and that the slopes of the $P_{\mathrm{n}}$ versus unloading rate plots are nearly constant. However, the $y$ intercept of the plots, i.e., the value of $P_{\mathrm{n}}$ as the unloading rate approaches zero, decreases as the peak load decreases. Figure 5 shows the variation between the $P_{\mathrm{n}}$ at zero unloading rate versus the peak load from the data in Fig. 4. It is evident that the $P_{\mathrm{n}}$ at zero unloading rate is always about $89.6 \%$ of the peak load.

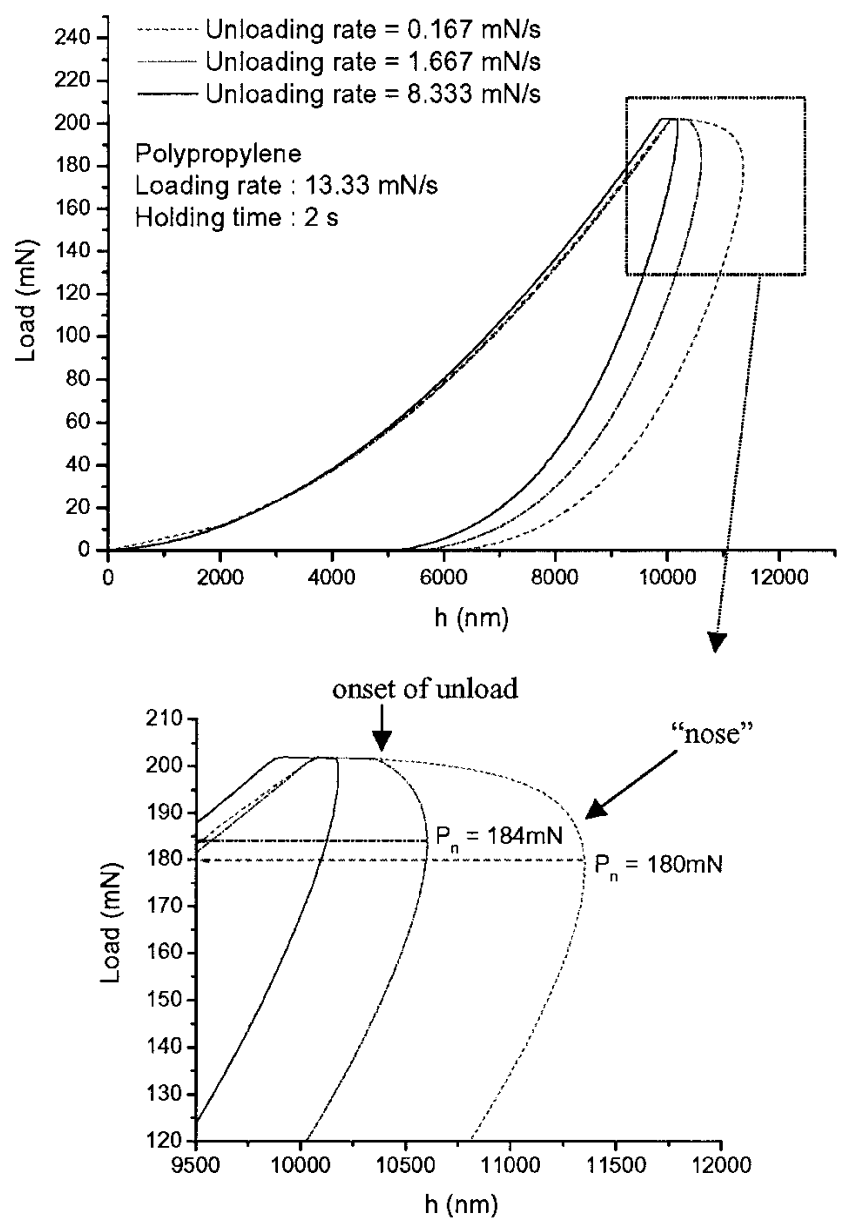

FIG. 2. Load versus displacement curves of PP at different unloading rates. All three experiments had the same loading rate to the same peak load and the same holding time before unload.
Whether a nose can appear at all in the unloading curve also depends on the peak load $P_{\max }$. Figure 6 shows the effect of $P_{\max }$ on the appearance of the nose. Here, a series of independent indentation experiments were performed on PP using different $P_{\max }$, but with the same loading and unloading rates, as well as the same holding time at the peak load. It can be seen that for $P_{\max }$ larger than about $40-50 \mathrm{mN}$, the unloading curves clearly exhibit a nose, while for $P_{\max }$ lower than $40 \mathrm{mN}$, the unloading curves evidently exhibit positive slopes all the way downward from the onset of unload. It is thus apparent that there exists a critical $P_{\max }$ of about $40-50 \mathrm{mN}$, below which no nose will happen.

Polypropylene at Room Temperature

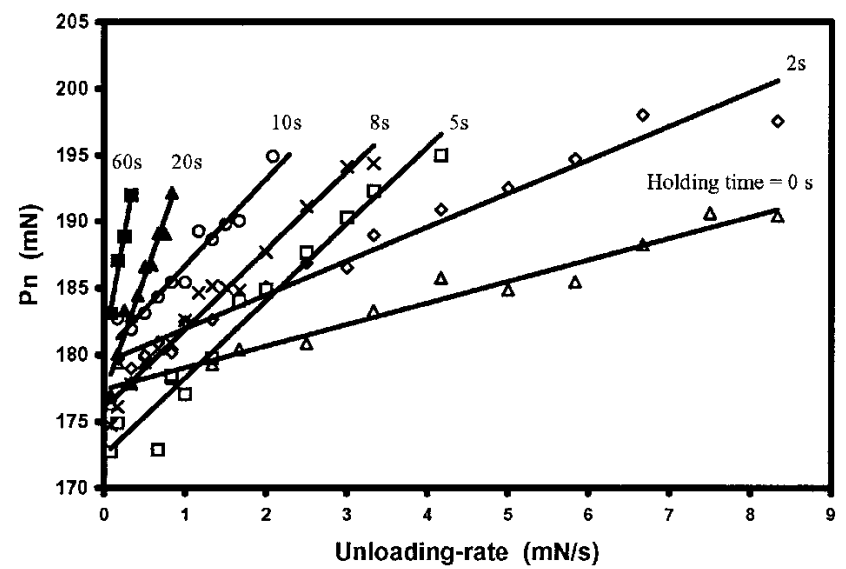

FIG. 3. Effect of unloading rate on the load $P_{\mathrm{n}}$ at which nose occurs in the load-displacement curve during unloading. All experiments were done with the same loading rate of $13.33 \mathrm{mN} / \mathrm{s}$ and the same maximum load of $200 \mathrm{mN}$, with different holding times at the maximum load, as shown.

Polypropylene at room temperature

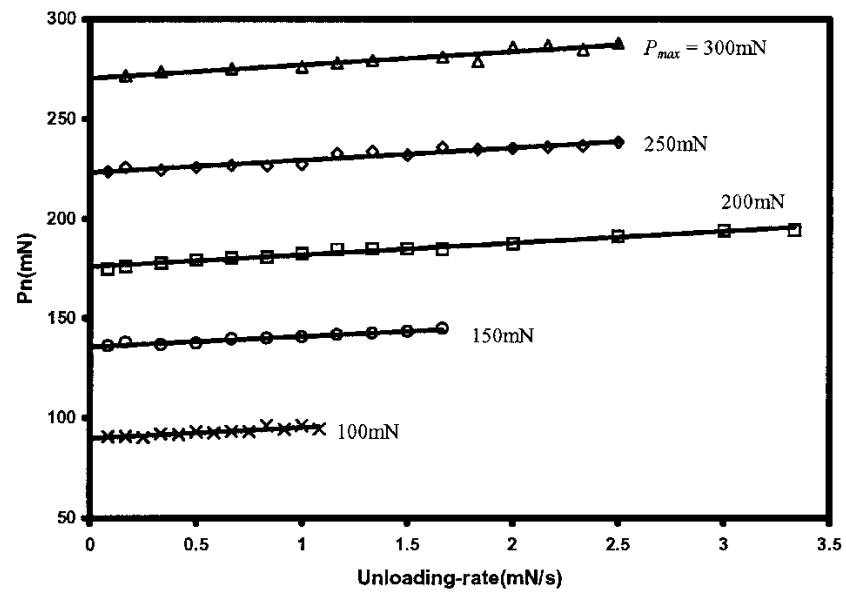

FIG. 4. Effect of unloading rate on the load $P_{\mathrm{n}}$ at which a nose occurs in the load-displacement curve during unloading at different peak loads. All experiments were done with the same loading rate of 13.33 $\mathrm{mN} / \mathrm{s}$ and the same holding time of $8 \mathrm{~s}$ at the maximum load. 


\section{DISCUSSION}

\section{A. Linear viscoelasticity analysis}

The above experimental results regarding the appearance of the nose in the unloading curve evidently require an explanation involving viscoelasticity. Here, we perform a linear viscoelastic analysis with the intent of predicting the conditions at which a nose will appear. The assumption of linearity here allows for an analytical solution and may be further justified if we limit our scope to a qualitative treatment aimed at capturing the physics of the problem. We therefore suppose the material to be indented is linear viscoelastic with stress-strain behavior represented by a Maxwell spring-dashpot model as shown in Fig. 7. Here, $G$ is the shear modulus of the material, and the viscosity component is represented by

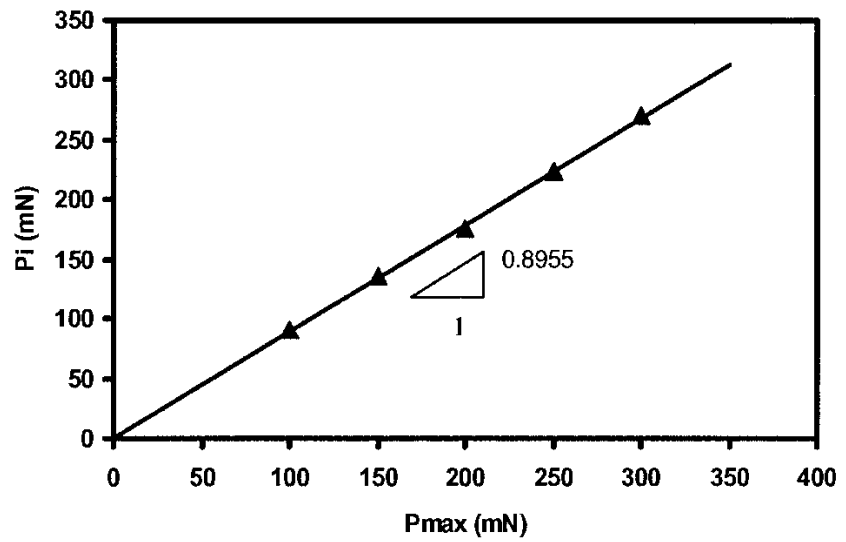

FIG. 5. Nose-load at zero unloading rate $\left(P_{\mathrm{i}}\right)$ versus $P_{\max }$. Data for $P_{\mathrm{i}}$ were taken from the $y$ intercepts in Fig. 4.

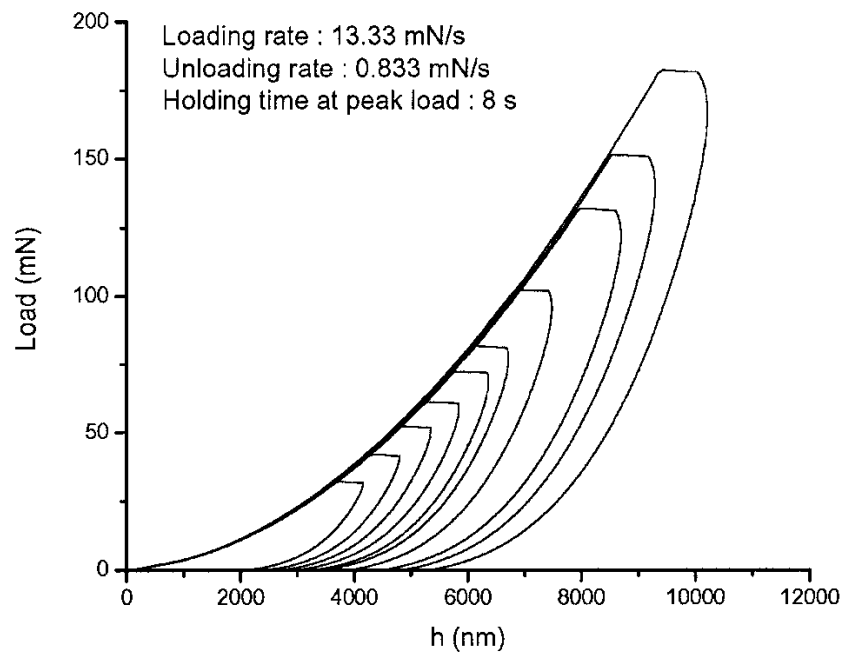

FIG. 6. Load-displacement curves for a series of independent indentation experiments on PP using the same loading and unloading rates and the same holding time at peak load but with different peak loads. Noses appear in the unloading curves for peak loads larger than about $40-50 \mathrm{mN}$. When the peak load is below $40 \mathrm{mN}$, no nose appears in the unloading curve.

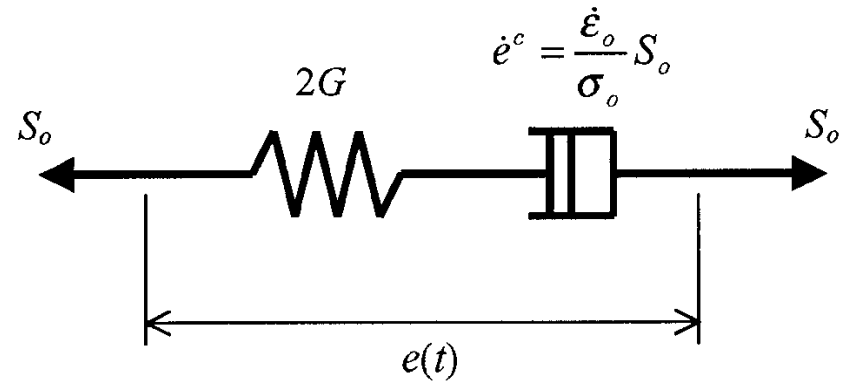

FIG. 7. Maxwell model for linear viscoelasticity. $S_{\mathrm{o}}$ is a deviatoric stress and $e$ the resultant deviatoric strain.

the dashpot with material parameters $\dot{\epsilon}_{\mathrm{o}}$ and $\sigma_{\mathrm{o}}$ with dimensions of strain rate and stress, respectively. The stress-strain relationship of the model in Fig. 7, expressed in terms of the deviatoric stress $S_{\mathrm{ij}}=\sigma_{\mathrm{ij}}$ $\delta_{\mathrm{ij}} \sigma_{\mathrm{kk}} / 3$ and deviatoric strain $e_{\mathrm{ij}}=\epsilon_{\mathrm{ij}}-\delta_{\mathrm{ij}} \epsilon_{\mathrm{kk}} / 3$, is

$$
\dot{e}_{\mathrm{ij}}=\frac{1}{2 G} \dot{S}_{\mathrm{ij}}+\frac{3 \dot{\epsilon}_{\mathrm{o}}}{2 \sigma_{\mathrm{o}}} S_{\mathrm{ij}}, \quad \sigma_{\mathrm{ii}}=3 B \epsilon_{\mathrm{ii}}
$$

where $B$ is the bulk modulus. Linear viscoelasticity problems are conventionally solved by the correspondence principle between elasticity and linear viscoelasticity as suggested by Radok. ${ }^{4}$ However, for the indentation problem shown in Fig. 1 in which the boundary also changes with load, Lee and Radok ${ }^{5}$ have shown that the correspondence principle can give a correct result only when the contact size $a(t)$ at time $t$ does not decrease. The indentation problem under a general load schedule has been solved by Ting, ${ }^{6}$ whose solution agrees with that of Lee and Radok $^{5}$ for the case of monotonically increasing $a(t)$.

Here we are interested in predicting the maximum in the $a(t)$ function, i.e., the nose, and so we can legitimately use the correspondence principle to calculate the increasing portion of the $a(t)$ function up to the maximum point. Such an analysis has been performed by Feng and Ngan, ${ }^{2}$ who showed that, for nondecreasing $a(t)$, the relation between the load $P$ and the contact size is given by

$$
\begin{aligned}
\frac{\dot{P}(t)}{E_{\mathrm{r}}} & +\frac{3 \dot{\epsilon}_{\mathrm{o}}}{4 \sigma_{\mathrm{o}}} P(t) \\
& +\frac{E^{2} \dot{\epsilon}_{\mathrm{o}}}{36 B^{2} \sigma_{\mathrm{o}}} \int_{0}^{t} \exp \left[-\frac{E \dot{\epsilon}_{\mathrm{o}}}{\sigma_{\mathrm{o}}}\left(t-t^{\prime}\right)\right] \dot{P}\left(t^{\prime}\right) \mathrm{d} t^{\prime}=2 a \dot{h},
\end{aligned}
$$

where $P(0)=0$. A straightforward calculation, assuming a load ramp followed by a load hold, shows that an upper bound of the ratio of the third term to the second term in Eq. (3) is $(1-2 v)^{2} / 3$, which is only $5.3 \%$ for Poisson's ratio $v=0.3$, or $1.3 \%$ for $v=0.4$. Hence, the third term in Eq. (3) is negligible compared to the second term. Equation (3) is valid for nondecreasing $a(t)$ or $h(t)$, and 
hence the condition $\dot{h}=0$ can be evaluated as a limiting case from it. Such a condition occurs at a load $P=P_{\mathrm{n}}$ given by

$$
\frac{\dot{P}}{E_{\mathrm{r}}}+\frac{3 \dot{\epsilon}_{\mathrm{o}}}{4 \sigma_{\mathrm{o}}} P_{\mathrm{n}}=0 \quad \text { or } \quad P_{\mathrm{n}}=\frac{4 \sigma_{\mathrm{o}}|\dot{P}|}{3 \dot{\epsilon}_{\mathrm{o}} E_{\mathrm{r}}}
$$

However, a nose in the unloading $P$ - $h$ curve will appear only when the $P_{\mathrm{n}}$ in Eq. (4) is smaller than the maximum load $P_{\max }$ from which the load decreases; i.e.,

$$
P_{\mathrm{n}}<P_{\max } \quad \text { or } \quad \frac{P_{\max }}{|\dot{P}|}>\frac{4 \sigma_{\mathrm{o}}}{3 \dot{\epsilon}_{\mathrm{o}} E_{\mathrm{r}}}
$$

In other words, a nose will appear only when the ratio $P_{\text {max }} /|\dot{P}|$ is larger than a critical value at a given temperature.

\section{B. Internal friction stress}

Equation (4) predicts that when a nose occurs, the load $P_{\mathrm{n}}$ at which it occurs is proportional to the unloading rate $|\dot{P}|$. However, the results in Figs. 3 and 4 show that $P_{\mathrm{n}}$ and $|\dot{P}|$ are only linearly related with an offset in $P_{\mathrm{n}}$ when $|\dot{P}| \rightarrow 0$. The experimental results therefore suggest that Eq. (4) should be modified into

$$
P_{\mathrm{n}}-P_{\mathrm{i}}=\frac{4 \sigma_{\mathrm{o}}|\dot{P}|}{3 \dot{\epsilon}_{\mathrm{o}} E_{\mathrm{r}}}
$$

where $P_{\mathrm{i}}$ is the constant offset load at zero unloading rate. We interpret $P_{\mathrm{i}}$ as due to the internal friction stress opposing slip, which was ignored in the linear viscoelasticity treatment leading to Eq. (4). The results in Fig. 3 indicate that the intercept load $P_{\mathrm{i}}$ does not seem to depend in a systematic manner on the holding time before unload, but the results in Figs. 4 and 5 indicate clearly that $P_{\mathrm{i}}$ is not a constant with respect to the maximum load $P_{\max }$, but is indeed always about $89.6 \%$ of $P_{\max }$. This means that the pressure under the indenter force $P_{\max }$, i.e., the hardness, will be proportional to $P_{\mathrm{i}}$ divided by the contact area. Since the internal stress should scale only with the applied pressure, our interpretation that $P_{\mathrm{i}}$ is due to the internal stress seems reasonable.

\section{Conditions for appearance of "nose"}

With the internal friction stress effect above, the condition in Eq. (5) for a nose to occur should be modified into

$$
\frac{P_{\max }-P_{\mathrm{i}}}{|\dot{P}|}>\frac{4 \sigma_{\mathrm{o}}}{3 \dot{\epsilon}_{\mathrm{o}} E_{\mathrm{r}}}
$$

Such a condition for a nose to occur is illustrated in Fig. 8. Recalling that in $\mathrm{PP}, P_{\mathrm{i}}=0.8955 P_{\max }$ from Fig. 5, Eq. (7) therefore becomes

$$
\frac{P_{\max }}{|\dot{P}|}>12.76 \times \frac{\sigma_{\mathrm{o}}}{\dot{\epsilon}_{\mathrm{o}} E_{\mathrm{r}}} .
$$

The results in Figs. 4 and 5 suggest that the slope of the $P_{\mathrm{n}}$ and $|\dot{P}|$ plot, at constant holding time at peak load, is not a constant but is an increasing function of the holding time. Since, from Eq. (6), the quantity $\sigma_{\mathrm{o}} / \dot{\epsilon}_{\mathrm{o}} E_{\mathrm{r}}$ is
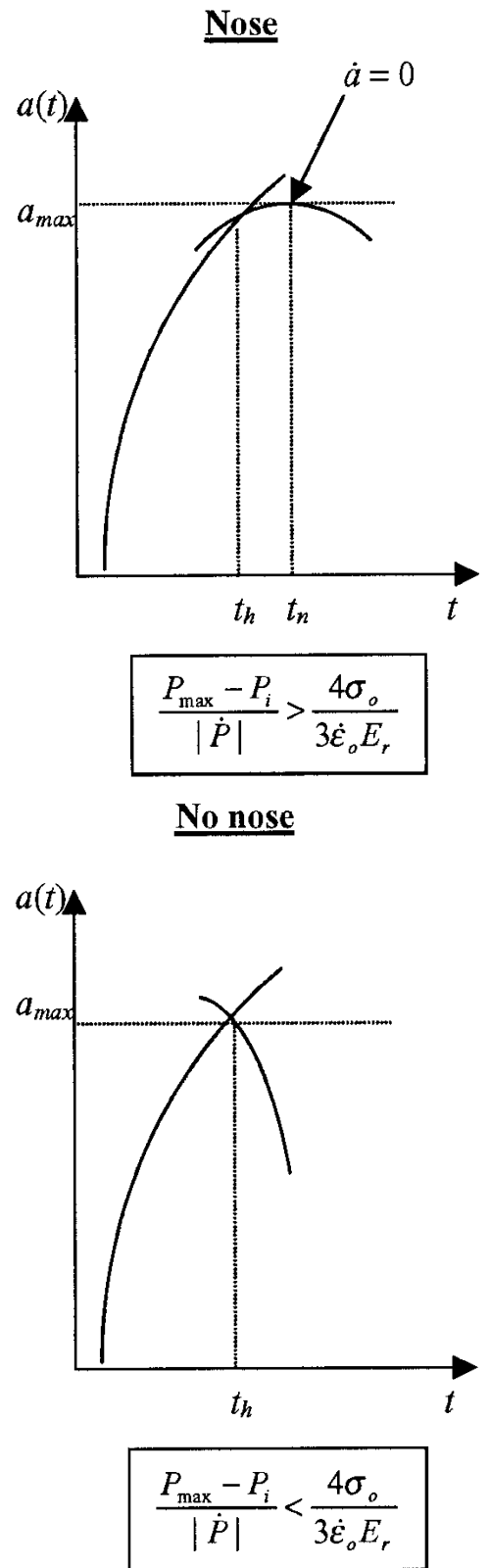

FIG. 8. Schematic showing the conditions at which a nose will or will not occur. The instant at which unload takes place is $t_{\mathrm{h}}$, the time instant at which a nose occurs is $t_{\mathrm{n}}$, and $P_{\mathrm{i}}$ is a constant due to the internal friction stress. 
proportional to the slope of the $P_{\mathrm{n}}$ and $|\dot{P}|$ plot, the right hand side of the inequality in Eq. (8) is therefore not a constant but is an increasing function of the holding time before unload. The reduced modulus $E_{\mathrm{r}}$ of our PP sample was measured in our experiments, using the Oliver-Pharr method with very fast unloading rate, to be about $2 \mathrm{GPa}$. [Taking the Poisson's ratio $v$ to be 0.4 for PP, the Young's modulus $E$ estimated from this value of $E_{\mathrm{r}}$, using the relation $E_{\mathrm{r}}=E /\left(1-v^{2}\right)$, is $1.7 \mathrm{GPa}$. This value of $E$ is in agreement with the range of 1.2 to $1.7 \mathrm{GPa}$ reported in the literature ${ }^{7}$ for PP.] Hence, from the slope of the $P_{\mathrm{n}}$ versus $|\dot{P}|$ plot in Fig. 3, the quantity $\dot{\epsilon}_{\mathrm{o}} / \sigma_{\mathrm{o}}$, termed here the viscosity parameter, can be calculated for each holding time, and the results are shown in Fig. 9. The observation here that the viscosity parameter changes with the holding time suggests that the structure of the PP polymer is changing during the hold at constant load.

Equation (8) therefore predicts that a nose will occur when (i) the unloading rate $|\dot{P}|$ is too slow, (ii) the holding time is too short so that $\dot{\epsilon}_{\mathrm{o}} / \sigma_{\mathrm{o}}$ is too large, or (iii) the peak load $P_{\max }$ is too large. Several sets of our present results provide quantitative checks for these conditions. First, when the best straight line for a holding time of $8 \mathrm{~s}$ in Fig. 3, for example, is extrapolated to $P_{\mathrm{n}}=P_{\max }$ $(200 \mathrm{mN})$, the $|\dot{P}|$ is about $4 \mathrm{mN} / \mathrm{s}$. This should correspond to the critical unloading rate beyond which no nose will occur. Using the relevant value of $E_{\mathrm{r}} \dot{\epsilon}_{\mathrm{o}} / \sigma_{\mathrm{o}}$ $\left(0.224 \mathrm{~s}^{-1}\right)$ for a holding time of $8 \mathrm{~s}$ from Fig. 9 , the critical unloading rate calculated from Eq. (8) is $3.5 \mathrm{mN} / \mathrm{s}$, which agrees well with the experimental value of $4 \mathrm{mN} / \mathrm{s}$. Secondly, Fig. 6 illustrates that at constant holding time and unloading rate, no nose will appear when the peak load $P_{\max }$ is below $40-50 \mathrm{mN}$. In Fig. 6,

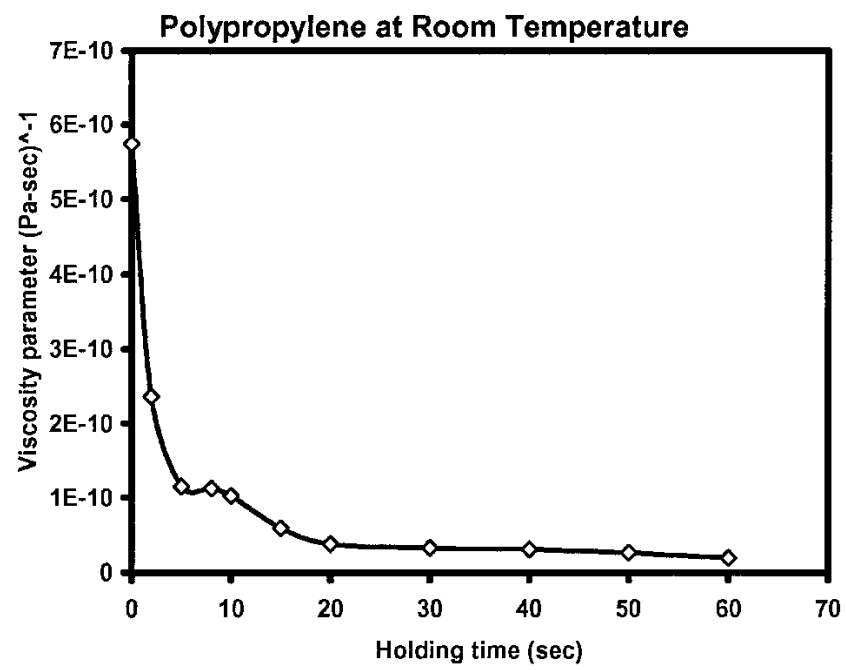

FIG. 9. Effect of holding time at peak load on the viscosity parameter, defined as $\dot{\epsilon}_{\mathrm{o}} / \sigma_{\mathrm{o}}$. The viscosity parameter is evaluated from the slope of the $P_{\mathrm{n}}$ versus $|\dot{P}|$ plots shown in Fig. 3 according to the Eq. (6). The reduced modulus $E_{\mathrm{r}}$ for PP is about $2 \mathrm{GPa}$. the unloading rate $|\dot{P}|$ is $0.833 \mathrm{mN} / \mathrm{s}$, and for the holding time of $8 \mathrm{~s}$ used, the value of $E_{\mathrm{r}} \dot{\epsilon}_{\mathrm{o}} / \sigma_{\mathrm{o}}$ from Fig. 9 is $0.224 \mathrm{~s}^{-1}$. Hence the critical $P_{\max }$ calculated from Eq. (8) is $47.5 \mathrm{mN}$, again in excellent agreement with the experimental observation in Fig. 6 .

An interesting further exercise would be investigation of the temperature dependence of the viscosity parameter using high-temperature indentation. The current results nevertheless suggest an alternative way for measuring the viscosity parameter of a material at the submicron length scale in addition to the established method of measuring the complex modulus by force modulation and noting the frequency response of the displacement in nanoindentation. ${ }^{8}$ More experiments on a wide range of materials would be required to determine whether the present approach can be developed into an established method for measuring viscoelastic properties, but there appears to be a superficial advantage of the present approach over the dynamic method using nanoindentation. In macroscopic tensile or torsional tests, it is well known that the complex modulus of a viscoelastic material is a strong function of the test frequency. For these macroscopic test geometries, it would be easy to construct simple spring-dashpot models to relate the measured complex modulus to the constitutive parameters. For example, if the Maxwell model shown in Fig. 7 is assumed, the loss modulus will approach the value $\sigma_{\mathrm{o}} \omega / \dot{\epsilon}_{\mathrm{o}}$ at very low frequency $\omega$, and the proportionality between the loss modulus and $\omega$ at low frequency has indeed been observed in many linear viscoelastic polymers. ${ }^{9}$ For the indentation geometry, however, it would be difficult to deconvolute constitutive parameters like $\dot{\epsilon}_{\mathrm{o}} / \sigma_{\mathrm{o}}$ from the frequency-dependent responses. Ting has shown that the solution to the problem of cyclic variation of the contact size during indentation is available only piecewise, and in any attempt to deconvolute material parameters from a measured cyclic response, one should fully consider Ting's solution. ${ }^{6}$

\section{CONCLUSIONS}

Using PP as a prototype viscoelastic material at room temperature, it was found that a nose may appear in the unloading $P$ - $h$ curve when (i) the unloading rate is small, (ii) the holding time before unload is short, and (iii) the maximum load is large. When a nose appears, the load at which it occurs is also found to decrease linearly with decreasing unloading rate. A linear viscoelasticity analysis, incorporating the Maxwell model, was performed to interpret this effect. The analysis, together with the assumption of the existence of an internal friction stress, predicts a linear variation between the nose load and the unloading rate, and the slope of such a linear variation was shown to be proportional to the viscosity parameter $\dot{\epsilon}_{\mathrm{o}} / \sigma_{\mathrm{o}}$ of the material. By fitting with the experimental 
results, the internal friction stress was found to be about $89 \%$ of the hardness of the PP material. The viscosity parameter $\dot{\epsilon}_{\mathrm{o}} / \sigma_{\mathrm{o}}$ in the linear analysis was found to be independent of the unloading rate and the maximum load, but it decreased as the holding time before unload increased.

The current results suggest a new method for measuring the viscosity parameter of a material at a given temperature. With the advent of high-temperature nanoindentation machines, the methodology proposed in this paper can enable submicron-scale creep properties of materials to be investigated as a function of temperature.

\section{ACKNOWLEDGMENT}

The work described in this paper was partially supported by a grant from the Research Grants Council of the Hong Kong Special Administrative Region, People's Republic of China (Project No. HKU 7062/01E).

\section{REFERENCES}

1. W.C. Oliver and G.M. Pharr, J. Mater. Res. 7, 1564 (1992).

2. G. Feng and A.H.W. Ngan, J. Mater. Res. 17, 660 (2002).

3. G. Feng and A.H.W. Ngan, in Fundamentals of Nanoindentation and Nanotribology II, edited by S.P. Baker, R.F. Cook, S.G. Corcoran, and N.R. Moody (Mater. Res. Soc. Symp. Proc. 649, Warrendale, PA, 2001), p. Q7.1.

4. J.R.M. Radok, Q. Appl. Math. 15, 198 (1957).

5. E.H. Lee and J.R.M. Radok, J. App. Mech. 27, 438 (1960).

6. T.C.T. Ting, J. App. Mech. 33, 845 (1966).

7. M.F. Ashby and D.R.H. Jones, Engineering Materials (Pergamon Press, Oxford, U.K., 1986), p. 31.

8. J.L. Loubet, W.C. Oliver, and B.N. Lucas, J. Mater. Res. 15, 1195 (2000).

9. J.D. Ferry, Viscoelastic Properties of Polymers (John Wiley \& Sons, New York, 1970), p. 45. 